\title{
"Yo me quedo en casa": Una experiencia de aprendizaje- servicio online en el Grado de Educación Infantil para ofrecer propuestas educativas a las familias
}

\section{Remedios Benítez}

\section{Laura Sánchez}

Universidad de Cádiz, España

\section{Resumen}

Se detalla una experiencia de aprendizaje-servicio online en el marco del último año del Grado en Educación Infantil de la Universidad de Cádiz. Experiencia mediada por la tecnología en unos tiempos de pandemia que nos han ofrecido la oportunidad de un nuevo aprendizaje, al reinventarnos. Partiendo de la necesidad social evidenciada por las diferentes familias en contextos y redes sociales desencadenó el diseño del mismo como respuesta a dicha necesidad. El grado de satisfacción del alumnado se evidencia en forma de voces, expresando lo aprendido mediante el mismo.

\section{Palabras clave}

Aprendizaje-servicio, voz del alumnado, tecnologías digitales, Educación Superior. 


\title{
'I am staying home': An online service-learning experience in the Early Childhood Education degree to offer educational proposals to families
}

\begin{abstract}
This paper discusses "Yo me quedo en casa" ('I am staying home'), an online servicelearning experience that was developed by final-year students of the Early Childhood Education degree at the University of Cadiz. The experience was mediated by technology in times of a pandemic, a situation that has forced us to reinvent ourselves and thus has offered us the opportunity for new learning. The service-learning project was designed as a response to the social needs shown by the different families in various contexts and social networks. The students who participated in the project voiced their level of satisfaction with the experience and highlighted what they had learned throughout the project.
\end{abstract}

\section{Keywords}

Service-learning, student voice, digital technologies, Higher Education. 


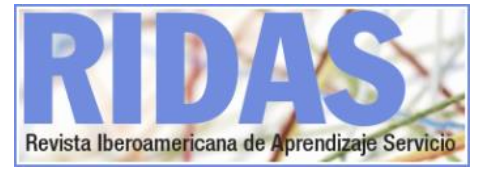

un compromiso (s.p.).

\section{Introducción}

Desde el curso académico 2013/2014 en la Universidad de Cádiz un grupo de docentes apostamos por la inclusión en nuestras aulas del aprendizaje-servicio como estrategia metodológica, en diferentes asignaturas que han constituido un itinerario curricular, permitiéndonos además estudiar e investigar diferentes aspectos relacionados con su desarrollo (Sánchez, Benítez, Quesada y García, 2019).

Consideramos esencial dejar constancia del enfoque y la concepción en la que nos postulamos, dentro de la infinidad de miradas existentes en la literatura, sobre aprendizaje-servicio. Según Benítez, García y Sánchez (2020):

Sostenemos una concepción del aprendizaje-servicio en la que el componente de "aprendizaje", del que participa el alumnado, incorpora el Trabajo por Proyectos (en adelante, TBP) y con ello sus características y sus fases, lo que nos aleja de una visión tecnificada del aprendizaje-servicio. Es desde aquí que nos permitimos apostar por la inclusión en la formación inicial del profesorado de propuestas orientadas al desarrollo de profesionales críticos a partir de aquellas que provocan el interés por conocer, comprender y actuar en el entorno como agentes transformadores de una realidad próxima, con la que se establece
Realizando proyectos reales con un impacto social (Martín et al., 2018) a la vez que una educación más inclusiva desde un aprendizaje por competencias (Puig, Gijón, Martín y Rubio, 2011).

En relación con el proyecto y la mediación con tecnologías de la información y la comunicación (TIC), el uso que habíamos hecho de las mismas era desde una integración básica, según la clasificación establecida por GarcíaGutiérrez, Ruiz-Corbella y Del Pozo (2020), dentro de los tres niveles de integración tecnológica en los proyectos de aprendizaje-servicio. Sin embargo, en esta experiencia que presentamos nos situamos en el nivel de inmersión tecnológica pues el desarrollo del servicio se hace íntegramente de forma virtual teniendo presente la intencionalidad educativa de las herramientas digitales. Compartiendo con García-Gutiérrez, Ruiz-Corbella y Del Pozo (2020), que:

Desde esta perspectiva, los proyectos de aprendizaje-servicio basados en tecnologías digitales nos permiten, además del desarrollo de las competencias digitales, ofrecer una apropiación ética y cívica de las mismas. En consecuencia, este tipo de proyectos nos permiten impulsar una visión humanista y humanizadora de las tecnologías digitales (p.72).

\section{Aportaciones de la tecnología}

Desde esa apropiación de las TIC se apuesta por defender la reflexión y la 
discusión crítica sobre la práctica, a la vez que fomentar el desarrollo profesional del profesorado, quien es considerado como orientador, crítico y transformador dentro del proceso tornándose como protagonista el estudiantado. Atribuyendo así mismo a las TIC competencias relacionadas con la transformación de las prácticas. El Consejo de la Unión Europea (2018) sugiere que la competencia digital es un uso seguro y crítico de las TIC de la Sociedad de la Información, en los ámbitos de trabajo, ocio y comunicación. Por lo que el profesorado y el alumnado necesitan construir dichas competencias para poder construir una ciudadanía que se actualice con los tiempos de la era digital. Dichas competencias, según el Marco Europeo para la competencia digital del profesorado -DigCompEdu(Instituto Nacional de Tecnologías Educativas y Formación del Profesorado, 2017) son 22 competencias englobadas en seis áreas que van desde el entorno profesional, a la creación de herramientas hasta facilitar la competencia digital del alumnado.

Es por ello por lo que el equipo docente debe permitirles la utilización de estas de forma mucho más profunda y creativa, proporcionándoles la experimentación e incluso transformando su contexto inmediato creando nuevas formas de contribuir, ofreciéndoles tecnologías de empoderamiento y participación (TEP) mejorando la calidad de sus aprendizajes (Martín, 2018). Todo ello desde un Diseño Universal para el Aprendizaje (DUA) que muestre disconformidad con el modelo existente, ofreciendo la respuesta a las necesidades y demandas del alumnado (Alba, Zubillaga, Sánchez, 2015).

\section{Aprendizaje-servicio online: experiencias en tiempo de pandemia}

La formación del aprendizaje-servicio en línea es conocida como eAprendizaje-servicio. El eAprendizajeservicio corresponde a una experiencia de aprendizaje-servicio con algún componente online, pudiendo ser la instrucción o el servicio (OrtegaRuipérez, 2020, 106).

A continuación, se ofrecen algunas aportaciones llevadas a cabo por diferentes organizaciones en España, donde los proyectos de aprendizajeservicio se han ido desarrollando de manera virtual, al igual que sucede con la que da nombre a este trabajo y que se centran en la situación insólita vivida a nivel mundial en estos últimos meses. Una gran mayoría han sido orientadas a romper la soledad provocada por el aislamiento de las personas enfermas, para ello se han realizado cartas, dibujos, llamadas telefónicas, cuentacuentos, materiales, pasteles, elementos simbólicos y campañas económicas para recaudar fondos para apoyar a las personas hospitalizadas. También han sido realizado trabajos de fomento de la lectura infantil, cápsulas musicales, fabricación de equipos de protección individua (EPIs), proyectos intergeneracionales, infografía a modo de guía básica para ayudar a la ciudadanía a conocer sus derechos durante el estado de alarma destinados a paliar consecuencias del COVID-19, 
ofreciendo de este modo posibles soluciones a situaciones reales.

También se encuentran proyectos que ya se habían iniciado antes de la pandemia, dirigidos a población vulnerable, cuidado y concienciación en relación al medio ambiente, o cualquier otra causa, que se han adaptado al distanciamiento físico, buscando acciones posibles de ser llevadas a cabo en situación de confinamiento, campañas de sensibilización y prevención en relación al COVID-19, creación de la piedra de la esperanza para las personas adultas mayores, apoyo escolar tanto a la infancia como a la docencia, proyectos de revitalización del tejido social y comercial de la población, creación de herramientas o dispositivos que se pueden al menos diseñar o en algunos casos, crear y distribuir aun estando en período de confinamiento, teniendo relación o no con dicha situación. Muchos de estos proyectos son colaborativos entre las familias, el profesorado y el alumnado generando alternativas a las dificultades creadas por el estado de alarma (Red Española de Aprendizaje-Servicio, 2020; Batlle, 2020).

\section{Descripción de la experiencia}

La experiencia ha tenido lugar durante el curso académico 2019/2020 en el marco de la asignatura Fundamentos Pedagógicos de las Necesidades Educativas en la Infancia. Esta asignatura, se desarrolla en el segundo semestre (de febrero a junio). Justo cuando comenzábamos a trabajar con los diferentes equipos en diversos proyectos que permitan construir los conocimientos y desarrollar competencias propias de la asignatura, se decretó el estado de alarma en nuestro país debido a la pandemia mundial provocada por el COVID-19. Ello trajo consigo un ajuste de toda la asignatura para adecuarnos, responder y transitar la nueva situación. Estos proyectos a lo que aludimos se desarrollan bajo la estrategia metodológica del aprendizaje-servicio como eje vertebrador de la misma.

El equipo pedagógico se coordinó para dar sentido, contenido y sostén emocional a lo que estaba aconteciendo desde los parámetros de una asignatura que fomenta, favorece y propicia la reflexión, el diálogo y el análisis crítico de la sociedad como aspecto necesario y fundamental en la educación superior desde la responsabilidad social universitaria (García y Cotrina, 2015). Lo primero que hicimos fue escuchar las voces del alumnado, para conocer y comprender cómo estaban viviendo la situación sobrevenida y qué aspectos de su alrededor estaban siendo llamativos y significativos para ellos y ellas.

En el foro de la asignatura se comenzó un debate donde el alumnado mostró preocupación por la infancia, comenzando un diálogo en relación con las necesidades que había detectado. Fue tomando relevancia y significado para el alumnado la carencia educativolúdica que parte de la población infantil podría estar viviendo. Tras el conocimiento, negociación y discusión de dicha necesidad, se decidió poner en marcha un proyecto de aprendizajeservicio que se realizaría eminentemente virtual. Constaba de las 
siguientes fases:

Figura 1. Fases del proyecto de aprendizajeservicio

$\begin{array}{ll}\begin{array}{l}\text { Análisis de la } \\ \text { realidad }\end{array} & \begin{array}{l}\text { Detección de la } \\ \text { necesidad }\end{array} \\ \text { - Situación de la } & \text {-Carencia } \\ \text { infancia durante el } & \text { educativo-lúdica } \\ \text { confinamiento } & \text { que parte de la } \\ & \text { población infantil } \\ & \text { podría estar }\end{array}$
viviendo

Desarrollo del ApS -Llamamiento

Celebración - Contando mi -Difusión del proyecto en la web de Facebook historia

-Batería de actividades -Difusión y reflexión

Fuente: elaboración propia

Para la creación, diseño y desarrollo del proyecto se crearon diferentes equipos base a los cuales se dio cobertura con espacios en Google drive,

BigBlueButton y foros de diálogo interno. Así mismo, se crearon salas virtuales para cada equipo con el fin de favorecer y potenciar uno de los ingredientes del aprendizaje cooperativo, el cara a cara (Pujolàs, 2008) y una sala común para la toma de decisiones, andamiajes y resolución de problemas que fueran surgiendo por parte de cada uno de ellos y que como grupo-clase se iban solventando de forma conjunta. Además se creó por parte de las docentes de la asignatura una página web colaborativa en Facebook (Fundamentos online) a la cual se dio acceso a un miembro de cada equipo para que pudiera gestionar las publicaciones en relación al servicio que habían realizado dentro del proyecto con la intención de ofrecerles autonomía y toma de decisiones en el proceso, ayudando a la construcción de diferentes competencias, no solo digitales sino actitudes colaborativas y solidarias, además de ajustes al DUA.

En esta experiencia participaron aproximadamente 145 estudiantes conformando equipos de 4 a 7 personas.

El diseño del proyecto de aprendizajeservicio contenía cuatro grandes actuaciones:

Figura 2. Fases del desarrollo del proyecto de aprendizaje-servicio

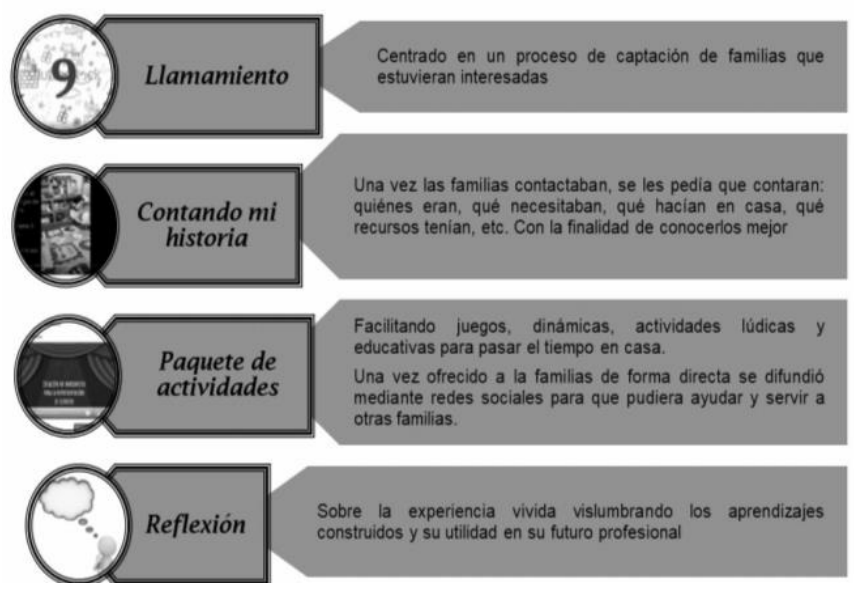

Fuente: elaboración propia

Para dar a conocer esta propuesta, el equipo pedagógico de la asignatura, con el fin de motivar al estudiantado, envió un vídeo grabado por las docentes en el que se ofrecía la oportunidad de pasar de un estado de letargo, miedo, confusión, incertidumbre, malestar, tristeza, 
angustia, incomprensión a un lugar de utilidad, solidaridad, actividad, compartir, motivación, ilusión, crecimiento y aprendizaje en el que sintieran lo que estaba sucediendo como una oportunidad de renacer, de reconvertirse, de resurgir, de rescatar las ganas, la ilusión y el interés que nos habían mostrada en día a día de clase y confiando es todo su potencial, respondiendo de este modo a los principios de Tomlinson (2005).

El proyecto comenzó con un estudio sobre la situación de partida, así como una llamada online, por medio de redes sociales, a las familias que pudieran tener la necesidad detectada (Ver figura 2). Se realizaron vídeos, imágenes, y diversas publicaciones de contenido. Se trataba de un mensaje trabajado y diseñado como equipo para atraer la atención de la familia en el formato que deseara hacerlo el equipo. Este mensaje iba dirigido a todas las familias del mundo, para que pudieran ponerse en contacto con los equipos y así estos lograran paliar su necesidad ajustándose a su realidad, recursos e intereses. El propósito de la llamada era que la familia que necesitaba enriquecer educativamente el confinamiento pudiera ponerse en contacto con los equipos de este aprendizaje-servicio online. Durante este proceso el equipo pedagógico de la asignatura preparaba y desarrollaba materiales y contenidos en relación con el DUA. La finalidad de dicho andamiaje era que el alumnado pudiese crear los materiales atendiendo a la diversidad, haciéndolos accesible a todas las personas

Tras recibir la respuesta al Ilamamiento de diversas familias comenzó lo que para el proyecto se denomina Contando mi historia: para conocer el día a día de las familias en casa con sus hijos y aproximarnos a su realidad. Podían realizarlo mediante la creación de un cuento, que decidían si querían compartir con otras familias. En caso de que así lo quisieran, se publicaba en redes sociales, convirtiéndose a su vez en un recurso para otras familias, siendo una primera actividad para las familias en la que aprendían según el asesoramiento de nuestro alumnado la creación de un cuento, sus partes, etc. lo que les permitía conocerse un poco mejor y ser un recuerdo creado por y para ellas con la que rememorar este momento excepcional y sin precedentes que estábamos viviendo.

Con el objetivo de que nuestro propio alumnado fuera aproximándose a la respuestas que deben ofrecer como futuros docentes a la diversidad que nos caracteriza como seres humanos, les propusimos un nuevo reto, en el que ajustar los cuentos o historias ofrecidas por las familias a los principios del DUA y así evitar posibles barreras para acceder a la información que contenían, al mismo tiempo que fortalece su compresión debido a que ofrece más de un canal y permite por tanto la complementariedad. Además, los equipos servían de apoyo y acompañamiento en la distancia para todo aquello que las familias necesitaran, por ejemplo: había familias que mandaban la historia contada y los equipos les hacían ilustraciones porque no se les daba bien dibujar o a la hora de redactar.

Tras recibir las historias de las 
diferentes familias, los equipos analizaban y estudiaban las aportaciones y todas las situaciones existentes, y decidían a qué familia darían respuesta. En la toma de decisiones consideraron analizar las posibilidades de las familias, para realmente atender a aquellas que tuvieran dicha necesidad. Además, se decidió por parte del equipo pedagógico que el diseño de actividades se hiciera a una familia concreta por dos motivos: el primero, que el alumnado entendiera la importancia de conocer una realidad concreta para darle respuesta desde los aportes de la atención a la diversidad y profundizar en la importancia de ofrecer a cada persona lo que necesita. Y el segundo, para evitar la saturación de los propios equipos y que realmente pudieran mantener un contacto y atención individualizada con la familia seleccionada, con la premisa de conocer para comprender, y que el trato y todo lo que acompañaba al servicio fuera esencialmente algo concreto para su propia realidad. Esto nos permitía a su vez, diferenciarnos de otros proyectos o plataformas virtuales que estaban ofreciendo recursos sin un trato individualizado y donde algunas veces las familias podrían encontrarse más trabas que ayuda. Como la intención final era compartir todo lo que se iba creando para la familia "apadrinada" siempre atendiendo a la diversidad respondiendo a los principios del DUA, las actividades diseñadas para una familia concreta eran subidas a la página web común del proyecto con el fin de que fuese un recurso extenso para otras familias y cada una encontrara en el citado espacio una amplia batería de propuestas y actividades que aunque no hubiesen sido creadas específicamente para ellas, les podían ser de utilidad. Esto es lo que llamamos difusión en la figura 1 .

Una vez pasadas las dos primeras actuaciones en el desarrollo del proyecto y seleccionada a la familia que iba a ser apadrinada, se invitaba al alumnado a reflexionar sobre posibles aprendizajes que iban a necesitar para dar respuesta al diseño de actividades, siempre teniendo en cuenta la realidad de la familia a la que estaban ofreciendo el servicio y los principios de la atención a la diversidad para que fuera de utilidad para otras familias.

Teniendo en cuenta las decisiones tomadas, cada miembro del equipo debía investigar y proponer al menos dos actividades educativas y didácticas desde lo que le gustaba hacer (música, manualidades, pintura, escribir, leer, deporte, etc.) y ofrecer alternativas a las familias (que lo necesitaban) con hijos de 3 a 5 años. Preparando un paquete de actividades, teniendo en cuenta los recursos que las familias podían tener en casa, puesto que no era posible salir de la misma. De este modo, atendíamos a otros tres de los ingredientes del aprendizaje cooperativo: la interdependencia positiva, la exigibilidad individual y las habilidades interpersonales y de trabajo en grupo (Pujolàs, 2008).

También se les invitaba a que en la variedad de actividades tuvieran en cuenta las inteligencias múltiples (Gardner, 2001) por lo que realizaban nuevas propuestas para atender y responder a los intereses planteados por las familias hacia otras actividades 
nuevas.

Para finalizar la cuarta actuación que contiene la figura 3, consistía en una nueva reflexión (otro de los ingredientes del aprendizaje cooperativo) por parte de los equipos para tomar conciencia y visibilizar los aprendizajes construidos, así como discurrir sobre las posibilidades y utilidades, tanto de los citados aprendizajes como de la experiencia vivida, que tiene para su futuro profesional y que algunos de ellos serán expuestos en líneas posteriores.

Llegamos a la última fase del proyecto, la celebración. Esta se llevó a cabo de dos formas distintas y a la vez, por un lado se le ofreció la voz a través de la web Fundamentos online a todas las personas participantes del proyecto y por otra, se creó un cuento que contaba el proceso del mismo a modo de cierre.

\section{A modo de cierre}

Las propias voces del alumnado nos muestran los beneficios, enunciados en forma de aprendizajes:

Figura 3. Nube de palabras

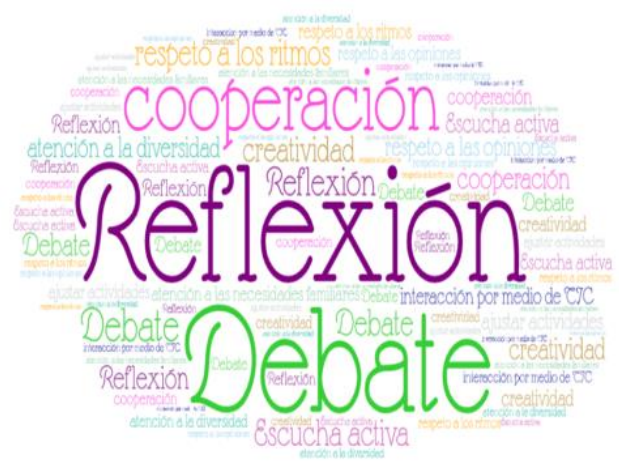

Fuente: elaboración propia
Vamos a centrarnos en aquellos aprendizajes relacionados con tres tópicos. Uno más general como son las competencias donde podemos identificar alusión a competencias de diversa índole; otro centrado en el eje principal de la asignatura, la atención a la diversidad y, por último, el ingrediente que hemos añadido a este curso, debido a la situación que nos encontrábamos, las TIC.

En cuanto a las competencias, el alumnado expresa que construye competencias básicas como la reflexión, la solidaridad, negociación, resolución de problemas:

- "Aprendizajes relacionados con la capacidad de observación, reflexión y análisis crítico de los problemas sociales, que hacen referencia a unas competencias que consideramos que debe dominar cualquier maestro $o$ maestra, pues estas permiten ampliar la mirada y comenzar a analizar la sociedad desde otra perspectiva" (A6).

- "Nos ha permitido desarrollar ciertas habilidades como la capacidad de escucha, la negociación, la capacidad de transmitir, entender $y$ comprender emociones $y$ sentimientos, y la capacidad de detectar problemas y buscar soluciones entre todos y todas las componentes del equipo, para así poder hacer un buen trabajo como docentes en un futuro" (A11).

Con relación a la atención a la 
diversidad, expresan la importancia del DUA, de ajustar las actividades, y la eliminación de estereotipos:

- "Hemos aprendido que es muy importante la forma que tenemos de comunicarnos con otras personas, es decir, atender a la diversidad ya que cada individuo tiene diferentes formas de acceder al aprendizaje, es decir, tiene diversos canales y formas de aprendizaje, los cuales hay que tener en consideración para proporcionar una educación inclusiva y de calidad. Por último, hemos aprendido de forma significativa lo importante que es ajustar el DUA al currículum así como a cualquier herramienta, material o metodología de trabajo para proporcionar una educación inclusiva para todos los niños y niñas" (B5).

- "Ha conseguido que seamos conscientes de la presencia en la sociedad de una serie de comportamientos estereotipados que como personas, como ciudadanos y sobre todo como futuras docentes hace que nos cuestionemos la eliminación de este tipo de actitudes lo antes posible con la finalidad de cambiar la mirada hacia la inclusión y la igualdad de oportunidades de todas las personas independientemente de las características, necesidades e intereses que tengan" (B3).

Sus aprendizajes sobre las TIC aparecen del siguiente modo, de una forma transformadora transitando entre las Tecnologías del Aprendizaje y el Conocimiento (TAC) y las TEP:

- "Nos hemos llevado bastantes aprendizajes y formación sobre las tic, esto de cara al futuro será un aprendizaje muy positivo ya que nos servirá como docentes para aplicarlo en nuestros centros o aulas" (B10).

- "Nos ha enseñado a utilizar las redes sociales para mostrar y compartir actividades enfocadas para los niños y niñas, pero que puedan disfrutar toda la familia. Nos ha aportado el tratar las redes sociales como un lazo que nos ha mantenido a todos unidos y conectados, y como una nueva forma de aprender" (B8).

- "A su vez, nuestra mirada hacia las redes sociales ha cambiado notablemente, pues más allá de ser un recurso lúdico y de ocio, es actualmente uno de los medios de difusión más importantes para llegar al mayor número de personas posible, convirtiéndose en una imprescindible ventana al mundo que puede hacer llegar nuestras voces a distintos lugares $y$ personas y recibir sus respuestas en consecuencia" (A8).

- "Progresivamente hemos aprendido a cooperar de forma más adecuada entre nosotras y nosotros, utilizando recursos como WhatsApp, Drive $y$ sesiones virtuales" (A10).

- "Ofrecer actividades a aquellas

Benítez, R. y Sánchez, L. (2020). Yo me quedo en casa: una experiencia de aprendizaje-servicio online en el Grado de Educación Infantil para ofrecer propuestas educativas a las familias. RIDAS, Revista Iberoamericana de Aprendizaje Servicio, 10, 23-35. DOI10.1344/RIDAS2020.10.3 
familias por medio de nuevas vías como son la de redes sociales a través de vídeo, por lo que hemos manejado el uso del formato vídeo" (A1).

- "Gracias a las circunstancias (viéndolo de forma positiva) hemos desarrollado y potenciado el manejo de las redes sociales y las nuevas tecnologías enfocadas a la comunicación eficiente" (A11).

Para finalizar nos gustaría ofrecer un aprendizaje que se torna al mismo tiempo como resultado del proyecto:

- "Uno de los aprendizajes más importantes que nos hemos llevado de este aprendizajeservicio, ha sido la relación con la familia, puesto que a pesar de la distancia (...) hemos sabido conectar con nuestra familia de referencia de forma correcta, conociendo todas sus necesidades, carencias y características para poder ofrecerle aquellas actividades $y$ recursos ajustados a ella. Consideramos que esta interacción con la familia es muy importante para el desarrollo integral del niño, y que durante el confinamiento hemos podido ayudarles para que todo fuera más ameno, además de permitir que el niño desarrolle su creatividad e imaginación con las actividades diseñadas para él" (B2).

Como puede apreciarse, el proyecto de aprendizaje-servicio online ha otorgado al alumnado protagonismo en todo el proceso, reconociendo sus aprendizajes como significativos y útiles, ayudándoles a comprender las problemáticas sociales reales, fomentando un desarrollo académico, cívico, vocacional, profesional, ético, moral, personal y social (Furco, 2007), y construyendo competencias explicitadas por el mismo como valiosas tanto personal como profesionalmente. Pudiendo afirmar que "en efecto, las tecnologías digitales facilitan el desarrollo de los proyectos de aprendizaje-servicio, a la vez que propician el desarrollo de proyectos novedosos que abren paso a nuevas modalidades de aprendizaje-servicio" (García-Gutiérrez, Ruiz-Corbella y Del Pozo, 2020, 71). Todo ello desde un diseño para todas las personas que propicie la participación y el acceso a la información.

\section{Referencias bibliográficas}

Alba, C., Zubillaga, A. y Sánchez, J.M. (2015). Tecnologías y Diseño Universal para el Aprendizaje (DUA): experiencias en el contexto universitario e implicaciones en la formación del profesorado. RELATEC - Revista Latinoamericana de Tecnología Educativa, 14(1), 89-100. doi: 10.17398/1695-288X.14.1.89

Batlle, R. (29 de abril de 2020). ApS y Coronavirus [Mensaje en un blog]. Recuperado de https://roserbatlle.net/aps-ycoronavirus/

Benítez, R. García, M. y Sánchez, L. (2020). Haciendo visible la línea invisible entre trabajo por proyectos y 
Aprendizaje-Servicio. En E. Caparrós, M. Gallardo, N, Alcaraz, N. y A.L. Rizzo. (Coord.), Educación inclusiva: un desafío y una oportunidad para la innovación educativa en la formación inicial del profesorado (en prensa)

Consejo de la Unión Europea (2018). Recomendación del Consejo, de 22 de mayo de 2018, relativa a las competencias clave para el aprendizaje permanente. Diario Oficial de la Unión Europea, 189/1 de 4 de junio de 2018.

Furco, A. (2007). Impacto de los proyectos de aprendizaje-servicio. En A. González (Coord.), Seminarios Internacionales de Aprendizaje y Servicio Solidario, (pp.175-183). Buenos Aires, Argentina: EUDEBA.

Gardner, H. (2001). Inteligencias múltiples. La teoría en la práctica. Barcelona, España: Paidós.

García, M. y Cotrina, M. (2015). El Aprendizaje y Servicio en la formación inicial del profesorado: de las prácticas educativas críticas a la institucionalización curricular. Revista Profesorado, 19(1), 8-25. Recuperado de:

https://recyt.fecyt.es/index.php/profes orado/article/view/41019

García-Gutiérrez, J., Ruiz-Corbella, M. y del Pozo, A. (2020). Innovación y aprendizaje-servicio virtual: elementos para una reflexión basada en la experiencia. RIDAS, Revista Iberoamericana de Aprendizaje Servicio, (9), 62-80. doi:10.1344/RIDAS2020.9.4

Instituto Nacional de Tecnologías
Educativas y Formación del Profesorado. (2017). Marco común de competencia digital docente. https://aprende.intef.es/sites/default/fil es/2018-05/2017 1020 MarcoCom\%C3\%BAn-de-CompetenciaDigital-Docente.pdf

Martin, M. V. (2018). ¿Consumidores o sujetos de derecho? La educación, entre las TIC y las TEP. Apuntes de Comunicación, Educación y Discurso, (2). doi:10.24215/25252046e009

Martín, E., Solari, M., De Vicente, J., Luque, M.J., Nieto, M. y Coll, C. (2018). La potencialidad del aprendizaje servicio para la personalización del aprendizaje escolar. RIDAS, Revista Iberoamericana de Aprendizaje Servicio, (5), 37-61. doi:10.1344/RIDAS2018.5.4

Ortega-Ruipérez, B. (2020). Formación del profesorado a distancia sobre aprendizaje-servicio: aspectos a considerar. RIDAS, Revista Iberoamericana de Aprendizaje Servicio, (9), 102-122. doi:10.1344/RIDAS2020.9.6

Puig, J., Gijón, M., Martín, X. y Rubio, L. (2011). Aprendizaje-servicio y educación para la ciudadanía. Revista de Educación, número extraordinario, 45-67. Recuperado de http://www.revistaeducacion.educacion .es/re2011/re2011 03.pdf

Pujolàs, P. (2008): Nueve ideas clave. El aprendizaje cooperativo. Barcelona, España: Graó.

Red Española de Aprendizaje-Servicio. (27 de marzo de 2020). ApS en tiempo 
de Coronavirus (I) [Mensaje en un blog]. Recuperado de

https://aprendizajeservicio.net/2020/03

227/aps-en-tiempos-de-coronavirus-i/

Sánchez, L., Benítez, R., Quesada, V. y

García, M. (2019). Competencias

emocionales en la formación inicial del

profesorado. El Aprendizaje y Servicio

como estrategia para su desarrollo.

Bordón, 71(3), 185-203.

doi:10.13042/Bordon.2019.68385

Tomlinson, C.A. (2005). Estrategias

para trabajar con la diversidad en el

aula. Buenos Aires, Argentina: Paidós. 